\title{
Touchless Palmprint and Finger Texture Recognition: A Deep Learning Fusion Approach
}

\author{
Angelo Genovese, Vincenzo Piuri, Fabio Scotti, Sarvesh Vishwakarma \\ Department of Computer Science, Università degli Studi di Milano, Italy. \\ firstname.lastname@unimi.it
}

\begin{abstract}
Biometric systems based on touchless and lessconstrained palmprint are being increasingly studied since they allow a favorable trade-off between high-accuracy and high usability recognition. Another advantage is that with a palmar hand acquisition, it is possible to extract the palmprint as well as the Inner Finger Texture (IFT) and increase the recognition accuracy without requiring further biometric acquisitions. Recently, most methods in the literature consider Deep Learning (DL) and Convolutional Neural Networks (CNN), due to their high recognition accuracy and the capability to adapt to biometric samples captured in heterogeneous and less-constrained conditions. However, current methods based on DL do not consider the fusion of palmprint with IFT. In this work, we propose the first novel method in the literature based on a CNN to perform the fusion of palmprint and IFT using a single hand acquisition. Our approach uses an innovative procedure based on training the same CNN topology separately on the palmprint and the IFT, adapting the neural model to the different biometric traits, and then performing a feature-level fusion. We validated the proposed methodology on a public database captured in touchless and less-constrained conditions, with results showing that the fusion enabled to increase the recognition accuracy, without requiring multiple biometric acquisitions.
\end{abstract}

Index Terms - Deep Learning, CNN, Palmprint, Finger

\section{INTRODUCTION}

Biometric systems are used to recognize individuals based on their physiological or behaviorial traits, without the need to remember passwords (that can be forgotten) or carry tokens (that can be stolen). The most widespread biometric systems consider physiological traits such as the fingerprint, palmprint, face, or iris, and behavioral traits such as the voice and signature [1].

Among physiological traits, the palmprint and the finger surface are being increasingly studied due to their favorable trade-off between high accuracy and high usability [2, 3, 4]. Recently, most biometric systems based on the palmprint capture the biometric samples using touchless acquisition procedures, where the surface of the hand does not touch any surface, therefore having high usability, positive social acceptance, and low intrusiveness $[3,5]$. In addition, it is often possible to capture both the palmprint and the inner finger surfaces with a single image of the hand, therefore enabling

This work was supported in part by the Italian Ministry of Research as part of the PRIN project COSMOS (201548C5NT). We gratefully acknowledge the support of NVIDIA Corporation with the donation of the Titan X Pascal GPU used for this research, within the project "Deep Learning and CUDA for advanced and less-constrained biometric systems".

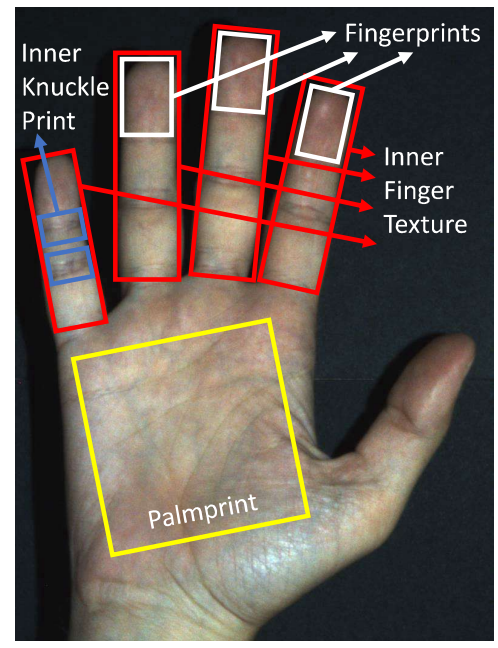

Figure 1. Position of the main biometric traits in a palmar acquisition of the hand. In this work, we consider the fusion of the palmprint and the Inner Finger Texture (IFT), which can be both extracted from the same palmar hand acquisition.

the use of a multimodal biometric system and increasing the recognition accuracy with a single biometric acquisition. In fact, the use of the inner finger surface in combination with palmprint has been proven to often increase the recognition performance [6].

Fig. 1 shows the position of the palmprint and finger areas in the context of hand-based biometric recognition. In this work, we focus on the palmprint and on the entire inner surface of the finger, referred to as "Inner Finger Texture" (IFT). In the literature, several works consider the Finger KnucklePrint (FKP) extracted from dorsal hand images, however in this work we consider only the inner finger surfaces since it is possible to extract them from the same palmar hand image as the palmprint. Other methods in the literature perform the biometric recognition by focusing on the Inner KnucklePrint (IKP), which represent a specific area of the inner finger surface. However, in this work, we focus on the entire inner surface of the finger [7].

Currently, the majority of methods for biometric recognition based on the palmprint or the finger surface are based on local texture descriptors or coding-based methods $[2,7,8]$. However, these approaches consist of handcrafted feature extraction techniques with parameters that may need to be 


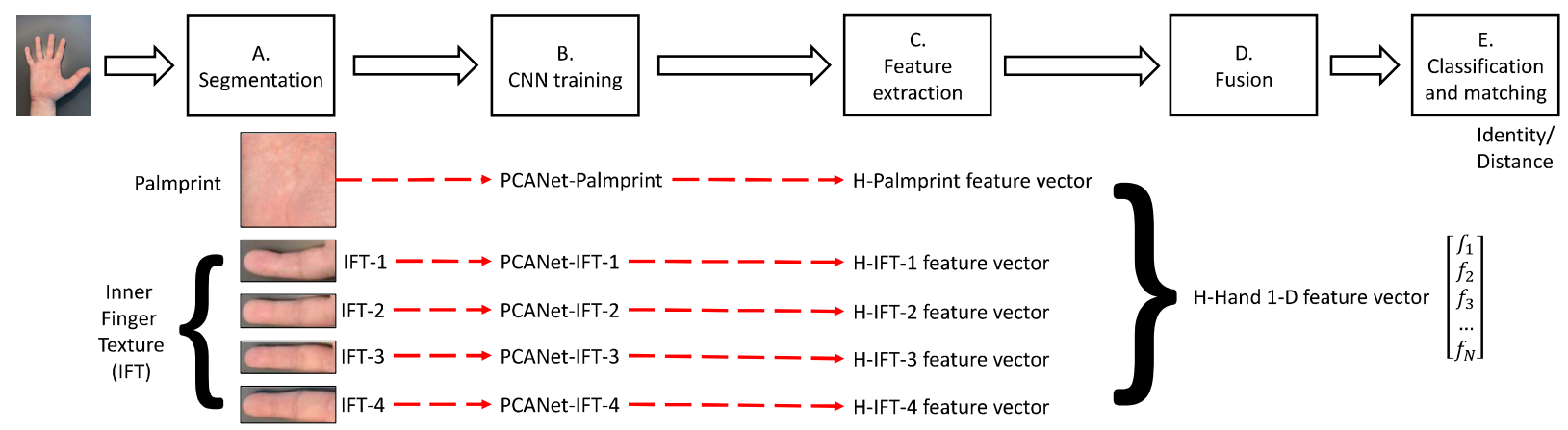

Figure 2. Outline of the proposed DL recognition method based on the fusion of palmprint and the IFT.

manually tuned for each different database to achieve the optimal recognition accuracy [9].

Recently, Deep Learning (DL) techniques such as Convolutional Neural Networks (CNN) have been increasingly studied in several application fields, including biometric recognition [10], due to their advantages of automatically learning data representations, thus not requiring a handcrafted feature extraction step, and adapting to biometric samples captured in heterogeneous conditions and with less-constrained acquisition procedures $[9,11,12]$. Several recent methods consider DL techniques for biometric recognition using touchless palmprint $[13,14,15,16]$ or using features based on the finger [17, $18,19,20]$. However, current DL methods for palmprint and finger recognition use supervised training procedures, extract FKP features from dorsal images of the hand, which require a different acquisition to capture also the palmprint, and do not consider the fusion of palmprint and IFT.

In this work, we propose the first novel method in the literature that uses a DL approach to perform the biometric recognition by fusing palmprint and IFT extracted from a single hand acquisition ${ }^{1}$. The proposed method has the following advantages: $i$ ) it uses a DL model that consists of a CNN trained using an unsupervised procedure, which does not require class labels; ii) it uses the same $\mathrm{CNN}$ model for all biometric traits, adapted separately to the palmprint and to the IFT extracted from the different fingers; iii) it adopts a featurelevel fusion of palmprint and IFT to increase the recognition accuracy, without requiring additional biometric acquisitions.

The paper is structured as follows. Section II introduces the relevant literature review. Section III describes the methodology. Section IV presents the experimental evaluation. Lastly, Section V concludes the paper.

\section{RELATED WORKS}

This section introduces the DL-based methods for palmprint and IFT recognition. In particular, it is possible to divide DLbased methods for palmprint recognition in three classes, based on the typology of the used CNN: $i$ ) methods based on CNNs pretrained on general purpose images; ii) methods based on

\footnotetext{
${ }^{1}$ The source code of the proposed method is available at http://iebil.di. unimi.it/fusionnet/index.htm.
}

CNNs with fixed filters; iii) methods using CNNs trained on palmprint images.

The methods in class i) use CNNs whose filters have been tuned by training the network on a general purpose database, such as the work described in [21], which presents a comparison of the different pretrained CNNs AlexNet, VGG16, and VGG-19. Similarly, the method presented in [13] uses a pretrained AlexNet to recognize newborns based on their palmprints.

The methods in class ii), rather than using filters pretrained on general purpose database, use fixed filters, such as the technique proposed in [22], which uses a CNN with filters based on the scattering transform [23].

The most accurate methods for DL-based palmprint recognition belong to class iii) and include the technique described in [15], which trains a CNN using a supervised procedure to optimize the separation between genuine and impostor comparisons. Contrarily to [15], the method presented in [24] performs the palmprint recognition by using the PCANet, trained using an unsupervised procedure based on PCA. The method proposed in [16] proposes the PalmNet, which introduces an adaptive methodology that tunes the Gabor filters to the palmprint samples.

To the best of our knowledge, the only method in the literature using a DL-based approach for biometric recognition using IFT is described in [17]. The method uses a CNN designed ad-hoc to process the IFT and trained using a supervised procedure to output the class of the corresponding individual.

The drawbacks of the considered DL-based methods for palmprint and IFT recognition consist in using either supervised training procedures, which require class labels corresponding to the individual during training, and not considering the fusion of the palmprint with IFT.

\section{Methodology}

The proposed approach uses an innovative procedure for the biometric recognition of the hand by performing a featurelevel fusion of the features computed by a DL model on the palmprint and on the IFT. In particular, in this work we consider the PCANet [24], a CNN trained using an unsupervised procedure based on PCA, applied on the palmprint and the IFT extracted from a single hand acquisition. We consider the 
(a)

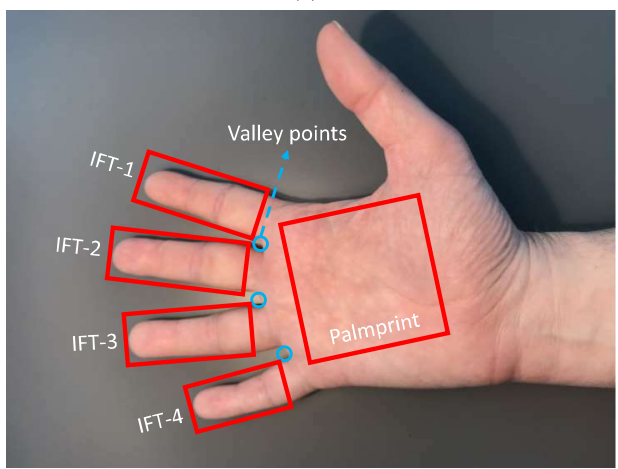

(b)

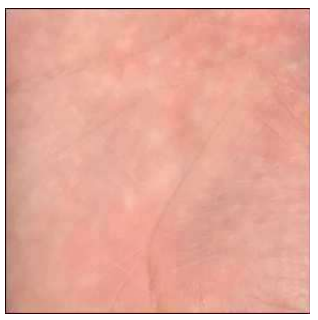

(c)

(d)

(e)

(f)

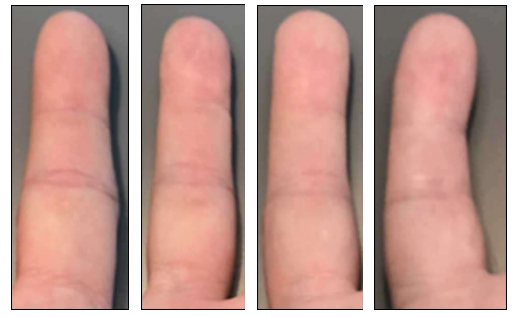

Figure 3. Example of the segmentation step to extract the Regions Of Interest (ROI) of the palmprint and the IFT based on the positions of the valley points. The output of the segmentation step consists of 5 images, one image for the palm and 4 images for each of the considered fingers: (b) palmprint; (c) IFT-1; (d) IFT-2; (e) IFT-3; (f) IFT-4.

PCA-based filters since they have been successfully applied for the biometric recognition based on different traits, such as palmprint and face $[14,25]$.

In this work, we apply the PCANet separately on the five biometric traits, consisting in the palmprint and the four IFT extracted from the index, middle, ring, and little finger, respectively. We consider the five biometric traits separately, instead of considering the whole image at the same time, since the PCANet requires the training images to be aligned [24]. By considering the whole image to train the PCANet, the four IFT would not be aligned due to differences in the relative positions of the fingers in different acquisitions. Therefore, we use a reference system based on valley points between the fingers to segment and then align the palmprint and the four IFT. For each biometric trait, the PCANet outputs a 1-D feature vector representing the biometric template. Then, we perform the feature-level fusion [26] of the resulting feature vectors to obtain a single biometric template for each hand acquisition. Lastly, we classify the obtained templates using a k-Nearest-Neighbors (k-NN) classifier with $k=1$ based on the Euclidean distance [27]. However, different classifiers and distance measures can be applied.

The method consists of the four following steps: A) segmentation, $B$ ) CNN training, $C$ ) feature extraction, $D$ ) fusion, and $E$ ) classification and matching. Fig. 2 presents the outline of the proposed method.

\section{A. Segmentation}

In the segmentation step, the method extracts the Regions of Interest (ROI) of the palmprint and the IFT. Fig. 3 presents

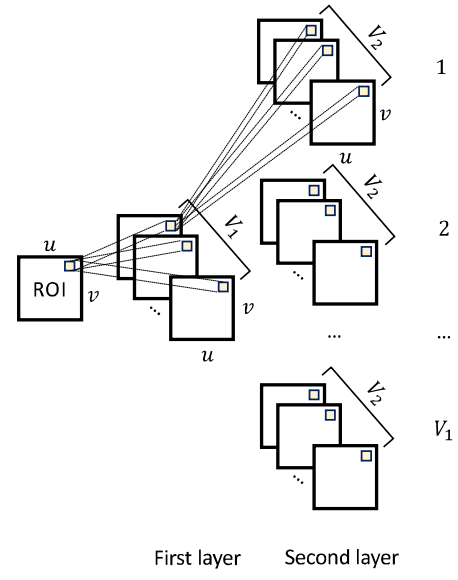

Figure 4. Topology of the PCANet used in the proposed approach. The same topology is separately applied to each different ROI computed in the segmentation step.

an example of the ROIs extracted from a hand acquisition.

To extract the ROI of the palmprint, we convert the image to grayscale and compute the binary mask of the hand by using a combination of the Kirsch edge detector [28] and Otsu's thresholding. Then, we extract the valleys between the fingers, corresponding to the intersection of the index, middle, and ring finger, by using a procedure based on the methods described in $[29,30,31]$. Lastly, we compute the ROI by using the reference system based on the valley points proposed in [5]. Fig. $3 \mathrm{~b}$ shows an example of a palmprint ROI.

To extract the IFT, we rotate the hand based on the reference system, to obtain an image of the hand with the fingers oriented leftwards, with the index finger at the top. Then, we select the 4 main connected components on the left of the palmprint ROI. For each connected component, we compute the orientation of the corresponding finger by analyzing the difference between the $x$ axis of the image and the major axis of the fitting ellipse. We compensate for the obtained orientation in each finger and then extract the region of the image enclosed by the bounding rectangle.

We resize the palmprint ROI to size $u \times v$ pixels and the IFT ROIs to size $a \times b$ pixels. Fig. 3c-f show examples of the ROIs of the IFT. In this work, we focused on hand images captured with a uniform background. However, the proposed method can be extended to hand images captured in-the-wild with unconstrained backgrounds, by using a different method to compute the binary mask, for example, based on skin-color thresholding [32] or CNNs [33].

\section{B. CNN Training}

In the CNN training step, we train a different PCANet for each different biometric trait extracted in the segmentation step described in Section III-A [24]. It is possible to divide the PCANet training procedure into three steps: $i$ ) collection of the local regions of the ROIs, ii) computation of the PCA and extraction of the filters of the $\mathrm{CNN}$, iii) construction of multiple layers of the $\mathrm{CNN}$. 
To train the PCANet based on $N$ ROI images, first, we extract the local regions $p_{i, j}$ centered in each pixel of the image. Each local region has dimensions of $m_{1} \times m_{2}$. We convert each region into a vector $\mathbf{p}_{\mathbf{i}, \mathbf{j}}$ and subtract the mean value from each vector. Then, we compute a matrix $P$ by concatenating the vectors $\mathbf{p}_{\mathbf{i}, \mathbf{j}}$ obtained from all the $N$ ROI images.

Second, we perform the PCA by extracting the $V_{1}$ principal eigenvectors from the matrix $P P^{T}$, obtaining $\mathbf{e}_{\mathbf{i}}$, with $i=$ $1,2, \ldots, V_{1}$. Then, we compute the $V_{1}$ filters of the first layer of the CNN by converting each eigenvector in a square matrix $E_{1, i} \in R^{m_{1} \times m_{2}}$.

Third, we compute a second layer by filtering the ROI with each of the filters $E_{1, i}$, obtaining $V_{1}$ images $F_{i}$. Then, we repeat steps $i$ )-ii) on the images $F_{i}$ and compute the filters $E_{2, j}$ of the second layer by extracting the $V_{2}$ principal eigenvectors of the PCA, with $j=1,2, \ldots, V_{2}$. We consider the trained PCANet as the ensemble of the computed filters: PCANet = $\left\{E_{1, i}, E_{2, j}\right\}$. Fig. 4 shows the topology of the PCANet.

We perform the training procedure on each different biometric trait in the hand image, obtaining 5 different PCANets: i) PCANet-IFT-1, ii) PCANet-IFT-2, iii) PCANet-IFT-3, iv) PCANet-IFT-4, v) PCANet-Palmprint.

\section{Feature Extraction}

In the feature extraction step, we apply each trained PCANet on the corresponding biometric trait, obtaining a 1-D feature vector for each trait. It is possible to divide the feature extraction step in three parts: i) image filtering using the PCANet, ii) image encoding, iii) template computation.

First, we filter the ROI using the $E_{1, i}$ filters of the first layer of the PCANet, obtaining $V_{1}$ images $F_{i}$. Then, we filter each of the images $F_{i}$ using the $E_{2, j}$ filters of the second layer of the PCANet, obtaining the $V_{1} \cdot V_{2}$ images $G_{k}$, with $k=1, \ldots, V_{1} \cdot V_{2}$. In particular, each set of $V_{2}$ images in the output of the second layer is obtained by filtering one of the $V_{1}$ images in the output of the first layer, as shown in Fig. 4. The sizes of the images remain constant during the PCANet processing.

Second, we binarize the $G_{k}$ images by applying to each pixel of all the images the following thresholding function:

$$
\operatorname{bin}(x)=\left\{\begin{array}{ll}
1 & \text { if } x>0 \\
0 & \text { otherwise }
\end{array},\right.
$$

obtaining the binary images $B_{k}$. Then, we consider separately each group of $V_{2}$ images. For each position $(x, y)$, we compute a binary vector by concatenating the binary bits, obtaining $\mathbf{b}=\left[B_{1}(x, y), B_{2}(x, y), \ldots, B_{V_{2}}(x, y)\right]$. We encode the binary vector as a decimal number, by using the following equation: $d=\sum_{m=1}^{V_{2}} 2^{j-1} \mathbf{b}(j)$. We perform the encoding for all positions $(x, y)$ to compute the decimal matrix $D(x, y)$ which encodes the result of the group of $V_{2}$ images. Then, we repeat the process for all $V_{1}$ groups and obtain the matrices $D_{i}(x, y)$, with $i=1,2, \ldots, V_{1}$.

Third, we construct the 1-D template $H$ by concatenating the blockwise histograms computed for each of the $n_{B}$ nonoverlapping blocks, with size $b_{1} \times b_{2}$, in the images $D_{i}$.
We compute the feature vector for each different biometric trait in the hand image, obtaining five feature vectors: $i) H$ IFT-1, ii) $H$-IFT-2, iii) $H$-IFT-3, iv) $H$-IFT-4, v) $H$-Palmprint.

\section{Fusion}

In the fusion step, we first perform the normalization of the feature vectors $H$ corresponding to the different ROIs. Then, we concatenate the normalized feature vectors to obtain the 1-D hand template $H$-Hand, using the following equation:

$$
H \text {-Hand }=\left[\begin{array}{c}
H \text {-Palmprint } \\
H \text {-IFT-1 } \\
H \text {-IFT-2 } \\
H \text {-IFT-3 } \\
H \text {-IFT-4 }
\end{array}\right] .
$$

In this work, we applied the min-max normalization, but different normalization schemes can be considered.

\section{E. Classification and Matching}

It is possible to use the proposed approach both in the identification and verification modalities [1]. In the identification modality, we perform a classification step using a k-NN classifier based on the Euclidean distance, with $k=1$ (denoted by $1-\mathrm{NN}$ in the following). We chose a $1-\mathrm{NN}$ classifier since it does not require training and has no parameters to tune. In this way, it is possible to evaluate the ability of the proposed approach to extract a highly discriminative template.

In the verification modality, we compute the distance $s$ between two hand templates $H$-Hand $\mathrm{H}_{1}$ and $H$-Hand $\mathrm{H}_{2}$ using a matching function $s_{1,2}=\operatorname{match}\left(H-\right.$ Hand $_{1}, H$-Hand $\left.{ }_{1}\right)$. In this work, we considered $\operatorname{match}(\cdot)$ as the Euclidean distance, however different distance measures can be considered.

\section{EXPERIMENTAL RESULTS}

This Section presents the experimental evaluation, by introducing the used database, presenting the error metrics, describing the tuning of the parameters, and then reporting the accuracy obtained using our approach. To evaluate the accuracy, we performed a technology evaluation [34] and compared the recognition performance of our method against other methods in the literature based on CNNs.

\section{A. Database}

To perform the accuracy evaluation, we considered the REgim Sfax Tunisia (REST) hand database 2016 [35], which contains 1945 palmprint samples captured from 358 individuals, whose ages range from 6 to 70 years. The acquisitions were performed using a low-cost digital camera under environmental indoor lighting, with frequent variations in the position and orientation of the hand. The segmentation step successfully extracted the ROIs of the palmprint and the IFT from 1937 samples, discarding only $\approx 0.5 \%$ of the samples.

\section{B. Evaluation and Error Metrics}

We evaluated the accuracy of the proposed approach by performing an $n$-fold cross-validation procedure [36]. In particular, we used $n=2$ and repeated the procedure 5 times [37]. One partition of the ROIs is used for training and the other partition for testing, with disjoint sets of individuals in the 
Table I

CLASSIFICATION ACCURACY ( $\%$ ) OF THE PROPOSED METHOD, WITHOUT THE FUSION STEP, COMPARED TO PRETRAINED CNNS.

\begin{tabular}{ccccccc}
\hline \multirow{2}{*}{ Ref. } & \multirow{2}{*}{ Method } & \multirow{5}{c}{ Classification accuracy (\%) } \\
& & Palm & IFT-1 & IFT-2 & IFT-3 & IFT-4 \\
\hline$[39]$ & AlexNet & 78.94 & 64.92 & 79.62 & 67.19 & 46.11 \\
{$[40]$} & VGG-16 & 66.83 & 58.36 & 74.53 & 60.28 & 36.99 \\
{$[40]$} & VGG-19 & 66.61 & 57.48 & 73.93 & 57.64 & 36.95 \\
- & PCANet & $\mathbf{9 3 . 0 3}$ & $\mathbf{7 2 . 0 7}$ & $\mathbf{8 7 . 6 3}$ & $\mathbf{7 7 . 6 9}$ & $\mathbf{5 8 . 0 9}$ \\
\hline
\end{tabular}

Table II

EER (\%) OF THE PROPOSED METHOD, WITHOUT THE FUSION STEP, COMPARED TO PRETRAINED CNNS.

\begin{tabular}{ccccccc}
\hline \multirow{2}{*}{ Ref. } & \multirow{2}{*}{ Method } & Palm & IFT-1 & IFT-2 & IFT-3 & IFT-4 \\
\hline$[39]$ & AlexNet & 13.16 & 11.39 & 8.92 & 10.39 & 15.00 \\
{$[40]$} & VGG-16 & 20.16 & 14.67 & 10.60 & 13.55 & 17.93 \\
{$[40]$} & VGG-19 & 19.30 & 13.38 & 11.40 & 13.07 & 18.15 \\
- & PCANet & $\mathbf{8 . 3 4}$ & $\mathbf{1 3 . 3 6}$ & $\mathbf{7 . 6 9}$ & $\mathbf{1 0 . 1 1}$ & $\mathbf{1 6 . 1 9}$ \\
\hline
\end{tabular}

training and testing subsets. We performed the feature extraction, fusion, classification, and matching steps on the testing subset. Then, we averaged the results on the 5 iterations.

We evaluated the performance of the proposed approach both in the identification and verification modalities. In the identification modality, we perform a classification using the 1-NN classifier based on the Euclidean distance, applied on the feature vectors computed from the testing subset. As the error measure, we consider the classification accuracy, expressed as the percentage of correctly classified samples. In the verification modality, we used the matching algorithm based on the Euclidean distance to compute the distances between the different templates. For each individual, we selected 4 templates as enrollment and the considered the other templates as testing [38]. For each comparison, we selected the final distance as the minimum distance between the test template and the enrollment templates. As the error measure, we considered the Equal Error Rate (EER).

\section{Parameter Tuning}

We chose the parameters of the proposed approach by performing an experimental evaluation with different possible values and we chose the values corresponding to the best recognition accuracy. In particular, we selected the numbers of filters of the CNN as $V_{1}=10$ and $V_{2}=10$, the size of the local regions $m_{1}=m_{2}=15$, and the size of the blocks used to compute the histograms as $b_{1}=b_{2}=23$. We chose the size of the palmprint ROI as $u=v=150$ and the size of the IFT ROI as $a=40, b=150$.

\section{Recognition Accuracy}

We compared the accuracy of the proposed method against pretrained CNNs used as feature extractors. In particular, we considered the pretrained AlexNet [39], VGG-16, and VGG19 [40] CNNs. For all three models, we used the features extracted from the sixth fully connected layer [21].

First, we evaluated the recognition accuracy of the proposed method without the fusion step, by applying the PCANet on the palmprint and the IFT separately. Table I shows the
Table III

ACCURACY COMPARISON OF FEATURE-LEVEL FUSION 1 AND FEATURE-LEVEL Fusion 2.

\begin{tabular}{|c|c|c|c|c|c|c|}
\hline \multirow[t]{2}{*}{ Method } & \multicolumn{2}{|c|}{$\begin{array}{c}\text { Palm } \\
\text { (no fusion) }\end{array}$} & \multicolumn{2}{|c|}{$\begin{array}{l}\text { Feature-Level } \\
\text { Fusion } 1\end{array}$} & \multicolumn{2}{|c|}{$\begin{array}{l}\text { Feature-Level } \\
\text { Fusion } 2\end{array}$} \\
\hline & Acc. $(\%)$ & EER (\%) & Acc. $(\%)$ & EER (\%) & Acc. $(\%)$ & EER $(\%)$ \\
\hline PCANet & 93.03 & 8.34 & 92.49 & 6.00 & 94.46 & 6.05 \\
\hline \multicolumn{7}{|c|}{ Notes: Acc. $=$ Classification accuracy. } \\
\hline \multicolumn{5}{|c|}{ 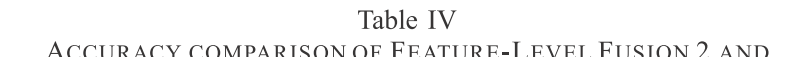 } & \multicolumn{2}{|c|}{$\begin{array}{l}\text { ACCURACY COMPARISON OF FEATURE-LEVEL FUSION } \\
\text { SCORE-LEVEL FUSION. }\end{array}$} \\
\hline \multirow[t]{2}{*}{ Method } & \multicolumn{2}{|c|}{$\begin{array}{l}\text { Palm } \\
\text { (no fusion) }\end{array}$} & \multicolumn{2}{|c|}{$\begin{array}{l}\text { Score-Level } \\
\text { Fusion }\end{array}$} & \multicolumn{2}{|c|}{$\begin{array}{l}\text { Feature-Level } \\
\text { Fusion } 2\end{array}$} \\
\hline & Acc. $(\%)$ & EER (\%) & Acc. $(\%)$ & $\operatorname{EER}(\%)$ & Acc. $(\%)$ & $\operatorname{EER}(\%)$ \\
\hline PCANet & 93.03 & 8.34 & 93.72 & 5.90 & 94.46 & 6.05 \\
\hline
\end{tabular}

obtained results expressed in terms of classification accuracy, while Table II contains the results in terms of EER. From the tables, it is possible to observe the proposed method based on PCANet outperforms the other methods based on CNNs. In addition, it is possible to observe that for some models the IFTs exhibit a comparable or superior accuracy than the palmprint, showing the validity in using the IFTs to increase the recognition performance.

Second, we analyzed the recognition accuracy of the proposed method with the fusion step. In particular, we compared the accuracy obtained by performing two different fusions:

- Feature-Level Fusion 1: IFT-1 + IFT-2 + IFT-3 + IFT-4;

- Feature-Level Fusion 2: Palmprint + IFT-1 + IFT-2 + IFT-3 + IFT-4.

Table III shows the obtained results for the Feature-Level Fusion 1 and Feature-Level Fusion 2. From the table, it is possible to observe that the proposed feature-level fusion increases the recognition accuracy with respect to using the palm and the IFTs separately. In particular, the proposed method based on Feature-Level Fusion 2 achieves the best classification accuracy. However, it is possible to notice that the fusion of the IFTs achieves accuracy comparable to using only the palmprint.

Lastly, we compared the accuracy of the proposed method based on Feature-Level Fusion 2 with the accuracy obtained using a Score-Level Fusion [41] of all five biometric traits based on a preliminary min-max score normalization and the sum rule [42]. From the table, it is possible to observe that the proposed method based on Feature-Level Fusion 2 achieves the greatest classification accuracy, showing its validity in the biometric identification modality. The proposed method based on Score-Level Fusion achieves the best EER, showing it would be the preferred choice in the verification modality.

\section{CONCLUSIONS}

In this paper, we proposed the first novel method in the literature based on DL for the fusion of the palmprint and the IFT using a single hand acquisition. The method is based on a novel use of the PCANet, a CNN trained using an 
unsupervised procedure, which is applied separately on the palmprint and on the IFT, and on a feature-level fusion of the resulting feature vectors.

We evaluated the proposed approach on a database of hand images captured with high variations in the position and orientation of the hand. The use of the proposed approach always allowed to increase the recognition accuracy of the biometric procedure, with respect to using the palmprint or the IFTs separately.

Future works should consider the use of segmentation algorithms to extract the hand even in images captured with unconstrained backgrounds, as well as other classifiers and different distance measures.

\section{REFERENCES}

[1] A. K. Jain, P. Flynn, and A. A. Ross, Handbook of Biometrics, 1st ed. Springer, 2010

[2] L. Fei, G. Lu, W. Jia, S. Teng, and D. Zhang, "Feature extraction methods for palmprint recognition: A survey and evaluation," IEEE Trans. Syst., Man, Cybern., Syst., pp. 1-18, 2018.

[3] A. Genovese, V. Piuri, and F. Scotti, Touchless Palmprint Recognition Systems, ser. Advances in Information Security, S. Jajodia, Ed. Springer, September 2014, vol. 60 .

[4] S. Barra, M. D. Marsico, M. Nappi, F. Narducci, and D. Riccio, "A hand-based biometric system in visible light for mobile environments," Inf. Sci., vol. 479, pp. 472-485, 2019.

[5] Ö. Bingöl and M. Ekinci, "Stereo-based palmprint recognition in various 3D postures," Expert Syst. Appl., vol. 78, pp. 74-88, 2017.

[6] G. K. O. Michael, T. Connie, and A. B. J. Teoh, "An innovative contactless palm print and knuckle print recognition system," Pattern Recognit. Lett., vol. 31, no. 12, pp. 1708-1719, 2010.

[7] G. Jaswal, A. Kaul, and R. Nath, "Knuckle print biometrics and fusion schemes - overview, challenges, and solutions," ACM Comput. Surv., vol. 49, no. 2, pp. 34:1-34:46, November 2016 .

[8] R. R. O. Al-Nima, S. S. Dlay, S. A. M. Al-Sumaidaee, W. L. Woo, and J. A. Chambers, "Robust feature extraction and salvage schemes for finger texture based biometrics," IET Biom., vol. 6, no. 2, pp. 43-52, 2017.

[9] R. Das, E. Piciucco, E. Maiorana, and P. Campisi, "Convolutional neural network for finger-vein-based biometric identification," IEEE Trans. Inf. Forensic. Secur., vol. 14, no. 2, pp. 360-373, February 2019.

[10] K. Sundararajan and D. L. Woodard, "Deep Learning for biometrics: A survey," ACM Comput. Surv., vol. 51, no. 3, pp. 65:1-65:34, May 2018.

[11] R. Donida Labati, A. Genovese, E. Muñoz, V. Piuri, and F. Scotti, "A novel pore extraction method for heterogeneous fingerprint images using Convolutional Neural Networks," Pattern Recognit. Lett., 2017.

[12] A. Anand, R. Donida Labati, A. Genovese, E. Muñoz, V. Piuri, and F. Scotti, "Age estimation based on face images and pre-trained convolutional neural networks," in Proc. of CISDA, 2017, pp. 1-7.

[13] R. Ramachandra, K. B. Raja, S. Venkatesh, S. Hegde, S. D. Dandappanavar, and C. Busch, "Verifying the newborns without infection risks using contactless palmprints," in Proc. of ICB, 2018, pp. 209-216.

[14] A. Meraoumia, F. Kadri, H. Bendjenna, S. Chitroub, and A. Bouridane, "Improving biometric identification performance using PCANet deep learning and multispectral palmprint," in Biometric Security and Privacy: Opportunities \& Challenges in The Big Data Era, R. Jiang, S. Almaadeed, A. Bouridane, D. Crookes, and A. Beghdadi, Eds. Cham: Springer, 2017, pp. 51-69.

[15] J. Svoboda, J. Masci, and M. M. Bronstein, "Palmprint recognition via discriminative index learning," in Proc. of ICPR, 2016, pp. 4232-4237.

[16] A. Genovese, V. Piuri, K. N. Plataniotis, and F. Scotti, "Palmnet: Gaborpca convolutional networks for touchless palmprint recognition," IEEE Trans. Inf. Forensics Security, 2019.

[18] Y. Zhai, H. Cao, L. Cao, H. Ma, J. Gan, J. Zeng, V. Piuri, F. Scotti, W. Deng, Y. Zhi, and J. Wang, "A novel finger-knuckle-print recognition based on batch-normalized CNN," in Proc. of CCBR, 2018, pp. 11-21.
[17] R. R. Omar, T. Han, S. A. M. Al-Sumaidaee, and T. Chen, "Deep finger texture learning for verifying people," IET Biom., vol. 8, no. 1, pp. 4048, 2019.

[19] R. Chlaoua, A. Meraoumia, K. E. Aiadi, and M. Korichi, "Deep learning for finger-knuckle-print identification system based on PCANet and SVM classifier," Evolving Systems, April 2018.

[20] G. Jaswal, A. Nigam, and R. Nath, "DeepKnuckle: revealing the human identity," Multimed. Tools Appl., vol. 76, no. 18, pp. 18955-18984, September 2017.

[21] A. S. Tarawneh, D. Chetverikov, and A. B. Hassanat, "Pilot comparative study of different Deep features for palmprint identification in lowquality images," CoRR, vol. abs/1804.04602, 2018.

[22] S. Minaee and Y. Wang, "Palmprint recognition using Deep Scattering Network," in Proc. of ISCAS, 2017, pp. 1-4.

[23] J. Bruna and S. Mallat, "Classification with scattering operators," in Proc. of CVPR, 2011, pp. 1561-1566.

[24] T. Chan, K. Jia, S. Gao, J. Lu, Z. Zeng, and Y. Ma, "PCANet: A simple Deep Learning baseline for image classification?" IEEE Trans. Image Process., vol. 24, no. 12, pp. 5017-5032, December 2015.

[25] C. Low, A. B. J. Teoh, and K. Toh, "Stacking PCANet+: An overly simplified convnets baseline for face recognition," IEEE Signal Process. Lett., vol. 24, no. 11, pp. 1581-1585, November 2017.

[26] A. Ross, "Fusion, feature-level," in Encyclopedia of Biometrics, S. Z. Li and A. Jain, Eds. Boston, MA: Springer, 2009, pp. 597-602.

[27] A. K. Jain, R. P. W. Duin, and J. Mao, "Statistical pattern recognition: a review," IEEE Trans. Pattern Anal. Mach. Intell., vol. 22, no. 1, pp. 4-37, January 2000.

[28] R. A. Kirsch, "Computer determination of the constituent structure of biological images," Computers and Biomedical Research, vol. 4, no. 3, pp. 315-328, 1971.

[29] T. Connie, A. B. J. Teoh, M. G. K. Ong, and D. N. C. Ling, "An automated palmprint recognition system," Image Vis Comput., vol. 23, no. 5, pp. 501-515, 2005.

[30] G. K. O. Michael, T. Connie, and A. B. J. Teoh, "Touch-less palm print biometrics: Novel design and implementation," Image Vis. Comput., vol. 26, no. 12, pp. 1551-1560, 2008.

[31] K. Ito, T. Sato, S. Aoyama, S. Sakai, S. Yusa, and T. Aoki, "Palm region extraction for contactless palmprint recognition," in Proc. of ICB, 2015, pp. 334-340.

[32] S. L. Phung, A. Bouzerdoum, and D. Chai, "A novel skin color model in $\mathrm{YCbCr}$ color space and its application to human face detection," in Proc. of ICIP, 2002.

[33] A. U. Khan and A. Borji, "Analysis of hand segmentation in the wild," in Proc. of CVPR, 2018, pp. 4710-4719.

[34] J. Jang and H. Kim, "Performance measures," in Encyclopedia of Biometrics, S. Li and A. Jain, Eds. Springer, 2009, pp. 1062-1068.

[35] N. Charfi, H. Trichili, A. M. Alimi, and B. Solaiman, "Local invariant representation for multi-instance touchless palmprint identification," in Proc. of SMC, 2016, pp. 3522-3527.

[36] R. O. Duda, P. E. Hart, and D. G. Stork, Pattern Classification (2Nd Edition). Wiley-Interscience, 2000.

[37] K. Nandakumar, Y. Chen, S. C. Dass, and A. Jain, "Likelihood ratiobased biometric score fusion," IEEE Trans. Pattern Anal. Mach. Intell., vol. 30, no. 2, pp. 342-347, February 2008.

[38] Y.-T. Luo, L.-Y. Zhao, B. Zhang, W. Jia, F. Xue, J.-T. Lu, Y.-H. Zhu, and B.-Q. Xu, "Local line directional pattern for palmprint recognition," Pattern Recognit., vol. 50, pp. 26-44, 2016.

[39] A. Krizhevsky, I. Sutskever, and G. E. Hinton, "ImageNet classification with Deep Convolutional Neural Networks," in Proc. of NIPS, 2012, pp. 1097-1105.

[40] K. Simonyan and A. Zisserman, "Very deep convolutional networks for large-scale image recognition," in Proc. of ICLR, 2015.

[41] A. Ross and K. Nandakumar, "Fusion, score-level," in Encyclopedia of Biometrics, S. Z. Li and A. Jain, Eds. Boston, MA: Springer, 2009, pp. 611-616.

[42] A. Anand, R. Donida Labati, A. Genovese, E. Muñoz, V. Piuri, F. Scotti, and G. Sforza, "Enhancing the performance of multimodal Automated Border Control systems," in Proc. of BIOSIG, 2016, pp. 1-5. 